\title{
Noninvasive evaluation of the migration effect of transplanted endothelial progenitor cells in ischemic muscle using a multimodal imaging agent
}

This article was published in the following Dove Press journal: International Journal of Nanomedicine

\author{
Xingui Peng' \\ Cong $\mathrm{Li}^{2}$ \\ Yingying $\mathrm{Bai}^{1}$ \\ Xinyi Wang' \\ Yi Zhang' \\ Yanli An' \\ Gao-Jun Teng' \\ Shenghong Ju' \\ 'Jiangsu Key Laboratory of Molecular \\ and Functional Imaging, Department \\ of Radiology, Zhongda Hospital, \\ Medical School of Southeast \\ University, Nanjing, ${ }^{2}$ Key Laboratory \\ of Smart Drug Delivery, Ministry of \\ Education \& PLA, School of Pharmacy, \\ Fudan University, Shanghai, People's \\ Republic of China
}

Background: Endothelial progenitor cells (EPCs) play an important role in repairing ischemia tissues. However, the survival, migration and therapeutic efficacy of EPCs after transplantation need to be better understood for further cell therapy.

Purpose: This study investigated the migration effect of EPCs labeled with a multimodal imaging agent in a murine ischemic hindlimb model, using magnetic resonance imaging (MRI) and optical imaging after transplantation.

Methods: EPCs derived from mouse bone marrow were labeled with a multimodal imaging agent and were administered through intracardiac delivery to mice with ischemic hindlimbs. The injected EPCs and their migration effect were observed via MRI and optical imaging in vivo, and then compared to a reference standard based on histological data. The quantification of gadolinium in tissue samples was done using inductively coupled plasma mass spectrometry (ICP-MS).

Results: Using in vivo MRI and optical imaging, the labeled EPCs were observed to migrate to ischemic muscle on days 3-5 after injection, while ex vivo, the EPCs were observed in the capillary vessels of the injured tissue. There were significant linear correlations between the Gd contents measured using ICP-MS in samples from the ischemic hindlimbs and livers and $\mathrm{T}_{1}$ relaxation times calculated using MRI, as well as the average fluorescence signal intensities recorded in optical images $\left(\mathrm{T}_{1}\right.$ relaxation time: $r=0.491$; average signal from optical imaging: $r=0.704, P<0.01$ ). EPC treatment upregulated the levels of $\mathrm{C}-\mathrm{X}-\mathrm{C}$ chemokine receptor 4 and vascular endothelial growth factor (VEGF) receptor 2 and enhanced the expression of stromal cell-derived factor-1 and VEGF.

Conclusion: Transplanted EPCs can be monitored with noninvasive MRI and optical imaging in vivo and were found to enhance the paracrine secretion of angiogenic factors.

Keywords: endothelial progenitor cell, ischemia, regeneration, cell tracking, magnetic resonance imaging, optical imaging

\section{Introduction}

Cell-based therapies are a promising therapeutic strategy for treating obstructive vascular diseases, which have become a major cause of mortality worldwide. ${ }^{1}$ Transplantation of EPCs, isolated from adult peripheral blood ${ }^{2}$ or derived from bone marrow, ${ }^{3}$ may lead to neovascularization in damaged tissues. ${ }^{4}$ Studies have shown that EPCs homing to ischemic tissues and organs do not always participate in the formation of neovasculature and may produce a variety of proangiogenic cytokines and growth factors to promote the proliferation and migration of preexisting endothelia cells. ${ }^{5-8}$ However, the existence and function of bone marrow-derived EPCs in adults are controversial and require further investigation. ${ }^{8}$ Therefore, noninvasive imaging methods capable of
Correspondence: Shenghong Ju Jiangsu Key Laboratory of Molecular and Functional Imaging, Department of Radiology, Zhongda Hospital, Medical School of Southeast University, 87 Ding Jia Qiao Road, Nanjing 210009 , People's Republic of China

Tel +862583272121

$\mathrm{Fax}+862583311083$

Email jsh0836@hotmail.com hereby accept the Terms. Non-commercial uses of the work are permitted without any furcther permission from Dove Medical Press Limited, provided the work is properly attributed. For permission for commercial use of this work, please see paragraphs 4.2 and 5 of our Terms (https://www.dovepress.com/terms.php). 
tracking transplanted EPCs and evaluating the survival and therapeutic efficacy of these cells in vivo would be useful for obtaining a better understanding of the processes and functions of transplanted EPCs in tissue repair and ultimately translating EPC therapy to clinical applications for treating diseases such as cardiac ischemia, ${ }^{9}$ vascular insufficiency, ${ }^{10}$ wound healing, ${ }^{11}$ and tumor inhibition. ${ }^{12}$

Among all available imaging modalities, MRI presents the advantages of a high spatial resolution, revealing soft tissue contrast and allowing 3D visualization of anatomic structures. However, this technique is limited by its low sensitivity when tracking transplanted cells. Nevertheless, optical imaging is a cost-effective imaging method that has recently emerged, with the advantages of high sensitivity and rapid acquisition. NIR imaging in the range of 650-900 nm exhibits a tissue penetration depth of approximately $1 \mathrm{~cm}$ and minimal background autofluorescence. ${ }^{13,14}$ Therefore, a combination of MRI and NIR optical imaging may represent a powerful tool for the imaging of transplanted cells in preclinical models with a high spatial resolution and sensitivity.

Visualization of cell migration in vivo using MRI can typically be achieved by labeling cells with MRI contrast agents, such as magnetic iron oxide nanoparticles or gadolinium-based contrast agents. ${ }^{15-18}$ More specifically, Gd-based contrast agents present the advantage of providing "bright" contrast enhancement, generated by shortening the longitudinal relaxation time of water molecules affected by the contrast agents; thus, these agents can be used to track cell migration and proliferation in vivo using $\mathrm{T}_{1}$-weighted imaging methods. In a previous study, a multimodality imaging probe, bCD-PLL, was developed for cell labeling and tracking. ${ }^{16,18}$ This probe comprised the paramagnetic $\mathrm{Gd}^{3+}$-DOTA chelator, the fluorescent molecule rhodamine and the NIR fluorophore Cy5.5, all of which were covalently attached to PLL, conjugated to a bCD as a carrier. In this study, the bCD-PLL was selected as the contrast agent to label EPCs because of its remarkable internalization efficiency via the clathrin-mediated endocytosis pathway. ${ }^{19}$ Here, we report the feasibility of in vivo tracking of EPCs labeled with the bCD-PLL imaging probe in a murine ischemic hindlimb model using MRI and optical imaging after intracardiac injection and the results of our investigation into the function of the injected EPCs.

\section{Materials and methods}

\section{Synthesis of the bCD-PLL imaging probe}

The multimodality imaging probe, bCD-PLL, was synthesized as previously described. ${ }^{20}$ Briefly, the bCD protein was isolated from transformed Escherichia coli transfected with a bCD cDNA vector. N-hydroxysuccinimide esters of DOTA, Cy5.5 and rhodamine were reacted with PLL, yielding a bCD-PLL-DOTA-Cy5.5-rhodamine complex. $\mathrm{Gd}^{3+}$ was subsequently incorporated into the complex via chelation with DOTA. The conjugation of bCD with the complex of PLL bound to $\mathrm{Gd}^{3+}$ DOTA and the NIR Cy5.5 and fluorescent rhodamine optical reporters yielded a multimodal imaging probe (Figure 1A). The molecular weight of bCD-PLL was $345 \mathrm{kDa}$, as measured using size-exclusion chromatography. ${ }^{20}$ An average hydrodynamic probe radius of $9.4 \mathrm{~nm}$ was determined by dynamic light scattering.

\section{Cell preparation and assessment}

This study was approved by the Institutional Animal Care and Use Committee of the Medical School of Southeast University (approval ID: SYXK-2010.4987), and followed the standards and requirements for the care and use of laboratory animals as set out by the committee. Male C57BLKS/J mice (Shanghai Laboratory Animal Center, Shanghai, People's Republic of China) were anesthetized with inhaled isoflurane (1.0\%-2.0\%; Shandong Keyuan Pharmaceutical Co., Ltd., Jinan, People's Republic of China) and subsequently sacrificed by cervical dislocation. Bone marrow-derived mononuclear cells were isolated from the tibias and femurs of the mice, and cultured as previously described. ${ }^{21}$ The collected cell pellet was suspended in growth factor-supplemented Endothelial Basal Medium-2 (EBM-2) medium (Lonza, Basel, Switzerland), followed by plating in flasks coated with fibronectin (Thermo Fisher Scientific, Waltham, MA, USA).

EPCs were analyzed after expansion in culture for 10-14 days. Assessment of the EPC phenotype was performed using immunofluorescence.

\section{Labeling EPCs with the bCD-PLL imaging probe}

The cultured EPCs were incubated with labeling medium containing bCD-PLL (bCD, $2 \mu \mathrm{M})$ in a flask for $24 \mathrm{~h}$. The labeled cells were subsequently washed three times with PBS to remove the free bCD-PLL. The labeling efficiency was determined based on the fluorescence signal from rhodamine using an inverted fluorescence microscope (Carl Zeiss, Jena, Germany). The details of this experiment have been previously described. ${ }^{16}$

\section{Mouse model of hindlimb ischemia and EPC transplantation}

To generate hindlimb ischemia, male athymic nude mice (15-20 g weight, 5 weeks of age) were anesthetized by isoflurane $(1.0 \%-1.5 \%)$ inhalation. The right femoral artery was exposed and excised from the proximal origin of the femoral 
A

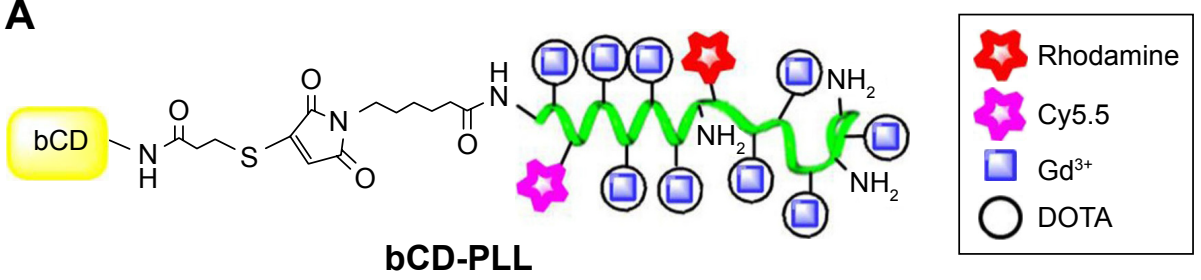

bCD-PLL

B

In vivo and in vitro EPC tracking by MRI, optical imaging, ICP-MS and immunohistofluorescence
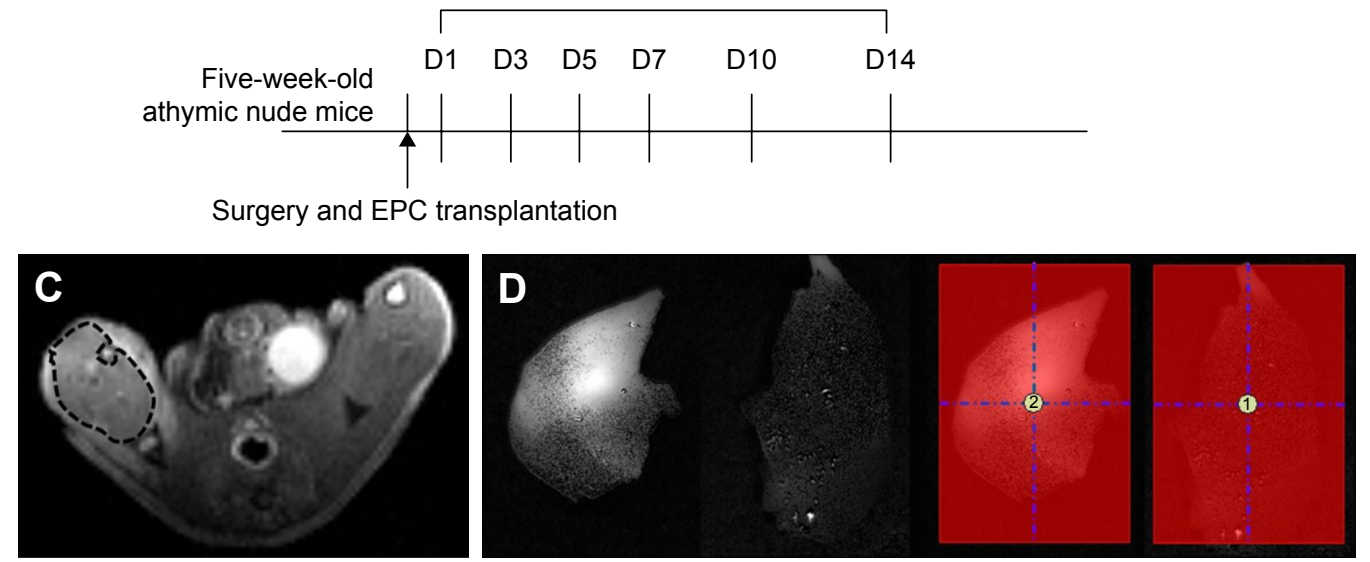

Figure I (A) Structure of bCD-PLL and (B) a diagram of the experimental protocol for tracking EPCs. (C) Example of ROls in MRI. $\mathrm{T}_{2}$-weighted images were used for

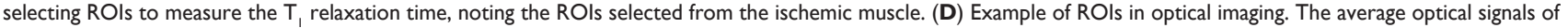
ischemic muscle and signal ratio (labeled EPCs/saline $=2 / \mathrm{I}$ ) were calculated from ROI analyses in two groups.

Abbreviations: bCD, bacterial cytosine deaminase; PLL, poly-L-lysine; EPC, endothelial progenitor cell; ROI, region of interest; MRI, magnetic resonance imaging; DOTA, tetraazacyclododecane-I,4,7,I0-tetraacetic acid; ICP-MS, inductively coupled plasma mass spectrometry.

artery to the bifurcation into the saphenous and popliteal arteries using an electrocoagulator, as previously described. ${ }^{22}$

At $24 \mathrm{~h}$ after the muscle ischemia procedure, the mice were randomly assigned to two groups for follow-up experiments involving different treatments, where the control group received saline without treatment, while the experimental group received EPCs labeled with the bCD-PLL probe. The EPCs labeled with the bCD-PLL probe $\left(1 \times 10^{6}, 150-200 \mu \mathrm{L}\right.$ each) and saline (150-200 $\mu \mathrm{L}$ each) were administered via intracardiac injection into the cavity of the left ventricle. Proper injection at the expected location was confirmed by observation of the pulsation of bright red blood backflowing into the syringe, as the left ventricle projects arterial blood.

\section{Tracking labeled EPCs after injection in vivo and in vitro}

The overall timeline of experimental procedures is outlined in Figure 1B. To track the injected EPCs labeled with the probe, MRI and optical imaging were performed on the two groups at 1,3, 5, 7, 10 and 14 days $(\mathrm{N}=6 /$ time point) after the injection of EPCs. Six mice from each group were sacrificed at each time point immediately after the imaging experiments for histological and quantification analyses.

\section{In vivo MRI scans}

All MRI experiments were performed at predetermined time points using a 7.0 T small animal MRI scanner (Bruker PharmaScan, ParaVision 5.1 software; Bruker, Billerica, MA, USA). The mice were placed in a supine position inside the coil prior to being placed in the scanner. They were anesthetized during scanning through the inhalation of $5 \%$ isoflurane. Their respiration rates were monitored using a physiological monitoring instrument (Bruker) for small animals.

The location of ischemic damage was determined using $\mathrm{T}_{2}$-weighted spin echo imaging with the parameters of TR and TE set at 3,000 and $36 \mathrm{~ms}$, respectively, and the number of excitations at 4, employing an FOV of $3.5 \times 3.5 \mathrm{~cm}$, a matrix of $256 \times 256$, a slice thickness of $1 \mathrm{~mm}$ and a total of 16 slices. For tracking the $\mathrm{Gd}^{3+}$-containing bCD-PLL probe, $\mathrm{T}_{1}$-weighted imaging was performed, with $\mathrm{TR}$ and $\mathrm{TE}$ of 500 and $15 \mathrm{~ms}$, respectively, employing the same FOV and slice locations as for $\mathrm{T}_{2}$-weighted imaging. The longitudinal relaxation time, $\mathrm{T}_{1}$, was determined by $\mathrm{T}_{1}$ relaxometry mapping, with the parameters including variable TRs of 5,000, $3,000,1,500,800,400$ and $200 \mathrm{~ms}$, TE of $11 \mathrm{~ms}$, a flip angle of $180^{\circ}$, an FOV of $3.5 \times 3.5 \mathrm{~cm}$, a matrix of $256 \times 256$ and a slice thickness of $1 \mathrm{~mm}$. The $\mathrm{T}_{1}$ relaxation times at each voxel 
were measured using the ParaVision 5.1 program (Bruker PharmaScan MRI) to generate $\mathrm{T}_{1}$ maps. ROIs were manually drawn in the ischemic gastrocnemius muscle (along the edge of the muscle) in the $\mathrm{T}_{2}$-weighted images, and as shown in Figure $1 \mathrm{C}$, these ROIs were copied and transformed to the same locations in $\mathrm{T}_{1}$ maps.

In vivo and ex vivo optical imaging for cell tracking In vivo optical imaging was performed using a Maestro Imaging System (CRi, Woburn, MA, USA) after MRI experiments at the same time points, and the mice were anesthetized, and subsequently placed in a prone and/or left-lateral position under the camera to maximally expose the injured limbs. Wavelengths of 576-621 nm (excitation) and $635 \mathrm{~nm}$ (emission) were used for detecting the Cy5.5 signal. Following the in vivo optical imaging experiments, the liver, kidneys, spleen, lungs and lower limbs (with the skin removed) were collected after the animals were sacrificed (n=6/group). Ex vivo optical images of the collected organs were obtained using the same parameters employed for imaging live animals.

The fluorescence images were unmixed based on the spectral patterns of the probe and autofluorescence using multispectral imaging capabilities and Maestro 2.10.0 software, implemented in an imager. ROIs were placed on the selected organs to analyze time-dependent signal changes (Figure 1D). The signals in each ROI were normalized by the exposure time and area of the ROI (scaled counts/s). ${ }^{23}$ The average optical signals from the ischemic muscle and the signal ratio (labeled EPCs/control) were calculated based on ROI analyses in the two groups.

\section{Immunofluorescence assessment of labeled EPCs}

Portions of the right gastrocnemius muscle samples ( $n=3$ /group) were frozen on dry ice and then sectioned in a cryostat at a thickness of $7 \mu \mathrm{m}$ onto PLL slides, for immunofluorescence labeling of cell markers in the EPCs. Frozen sections of ischemic adductor muscles were incubated using $10 \mu \mathrm{g} / \mathrm{mL}$ rabbit monoclonal anti-mouse CD34 (Santa Cruz Biotechnology Inc, Dallas, TX, USA), CD133 (Abnova, Taipei, Taiwan), VEGF receptor 2 (Abcam, Cambridge, UK) and CXCR4 (Abcam) antibodies followed by staining with an Alexa Fluor 488 goat anti-rabbit polyclonal antibody (Thermo Fisher Scientific). Selected sections were examined with confocal microscopy (LEXT; Olympus, Tokyo, Japan) to visualize the colocalization of rhodamine and these markers.

\section{Western blot analysis}

Total proteins were extracted from homogenized ischemic muscle tissue samples collected after imaging experiments.
The protein expression of VEGF receptor 2, VEGF, CXCR4 and SDF-1 was assessed by western blot analysis as previously described. ${ }^{24}$

\section{Quantification of Gd in tissue samples via ICP-MS}

The concentrations of Gd in the liver, kidneys, spleen and lungs as well as ischemic and nonischemic gastrocnemius muscle collected at each time point $(n=3)$ were analyzed using ICP-MS (ELAN9000; PerkinElmer, Waltham, MA, USA). ${ }^{18,24}$ Fresh liver, kidney, spleen and lung tissues as well as ischemic (right hindlimb) and nonischemic (left hindlimb) gastrocnemius muscle tissues were weighed (approximately $100 \mathrm{mg}$ per sample) and digested with nitric acid and hydrogen peroxide. After the tissue was fully dissolved, the sample was diluted to the proper concentration range for measurement of the ${ }^{157} \mathrm{Gd}$ concentration. ICP-MS was carried out using the following parameter settings: plasma power of $1,230 \mathrm{~W}$, cooling gas flow of $13.0 \mathrm{~L} / \mathrm{min}$, nebulizer gas flow of $0.9 \mathrm{~L} / \mathrm{min}$, nebulizer sample orifice of $1.0 \mathrm{~mm}$ and conical skimmer orifice of $0.7 \mathrm{~mm}$.

\section{Statistical analysis}

All statistical analyses were performed using SPSS software (SPSS for Windows, version 11.0, 2001; SPSS, Chicago, IL, USA). Numerical data were reported as the mean values \pm standard deviation. For statistical comparisons, independentsample $t$-tests, paired-sample $t$-tests and regression analysis were applied. A $P$-value of less than 0.05 was considered statistically significant.

\section{Results \\ Phenotype and endothelial characteristics of bone marrow-derived EPCs}

The shape of bone marrow-derived mononuclear cells changed from flat on day 3 to spindle-like (Figure 2A) after culturing for 7 days (Figure 2B). Co-staining with fluorescein isothiocyanate-UEA-1 and Dil-labeled acLDL showed that these cells exhibited characteristics of an endothelial cell phenotype, including the uptake of acLDL and binding of endothelial cell-specific lectin UEA-1 (Figure 2C). Immunofluorescence staining for CD34, CD133, VEGF receptor 2, vWF, CD146 and CD31 showed that these cells were positive for these stem cell and endothelial cell markers (Figure 2D). Staining for CXCR4, a marker of hematopoietic stem cells and progenitor cells, was also positive.

After incubation with the imaging probe (bCD-PLL, $2 \mu \mathrm{M}$ ) for $24 \mathrm{~h}$, the fluorescence signal of the rhodamine within the probe was detected in the perinuclear region of the cells (Figure 2E). The labeling efficiency of bCD-PLL 


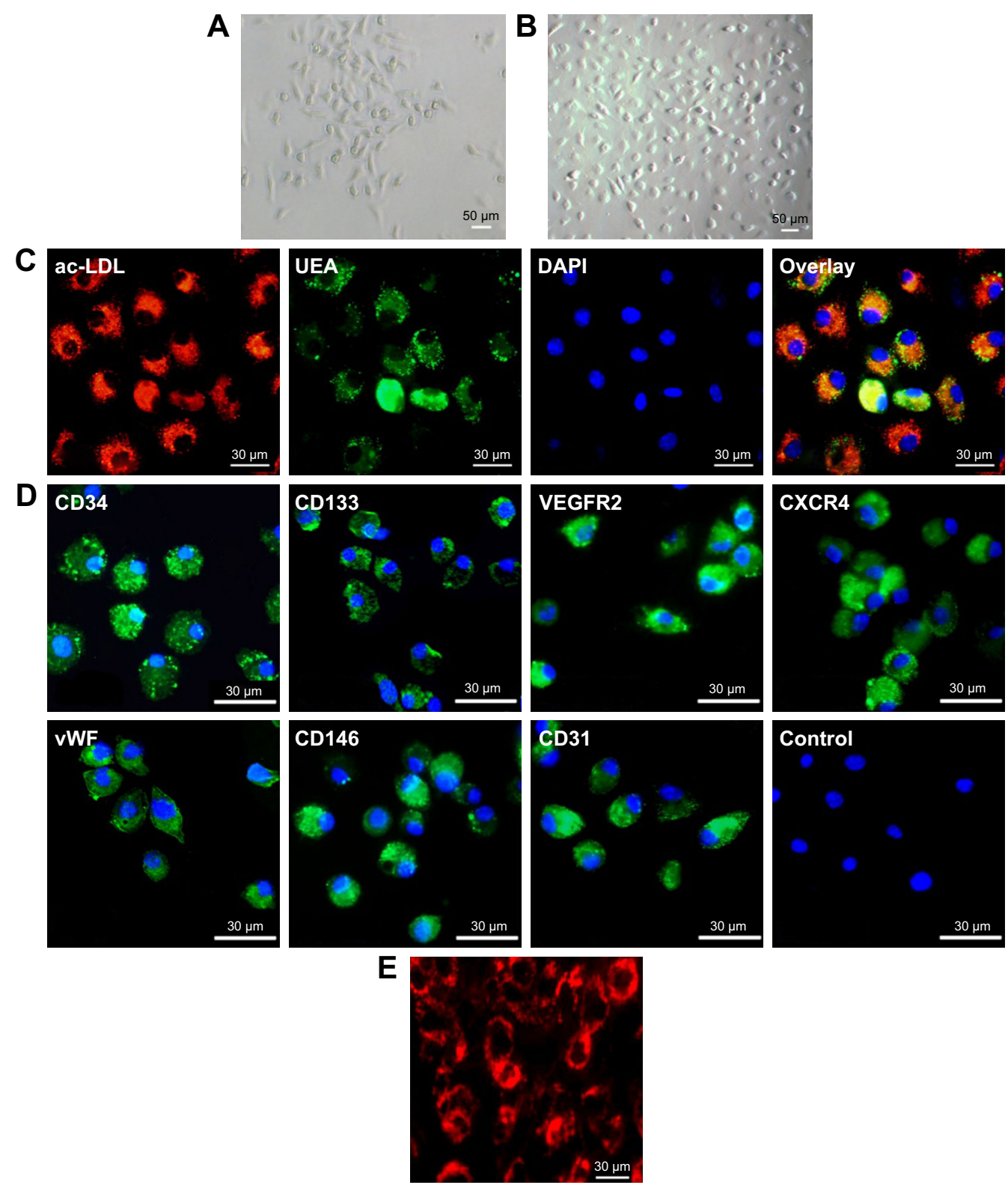

Figure 2 Morphological and immunocytochemical analyses of mouse bone marrow-derived EPCs. (A) The shape of mononuclear cells was thin and flat on day 3, and a round and fusiform appearance was subsequently observed on day 7 (magnification $\times 100$ ). (B) On day 14, the cells exhibited a typical fusiform or "cobblestone" morphology (magnification $\times 100$ ). (C) Uptake of Dil-labeled acLDL and binding of endothelial-specific fluorescein isothiocyanate-labeled lectin in EPCs in culture for I4 days (magnification $\times 400$ ). Lectin and lipoprotein positivity colocalized in $>95 \%$ of cells. (D) Immunocytochemical images of cells showing positive staining for the CD34, CDI33, VEGF receptor 2, CXCR4, vWF, CDI46 and CD3I markers. (E) Fluorescence image showing the uptake of multimodal imaging of bCD-PLL by EPCs (day I4) with positive rhodamine signals (magnification $\times 400$ ).

Abbreviations: EPC, endothelial progenitor cell; acLDL, acetylated low-density lipoprotein; VEGF, vascular endothelial growth factor; CXCR4, C-X-C chemokine receptor 4; vWF, von Willebrand factor; bCD, bacterial cytosine deaminase; PLL, poly-L-lysine; UEA, Ulex europaeus agglutinin-I; DAPI, 4',6-diamidino-2-phenylindole; VEGFR2, VEGF receptor 2 .

was approximately $100 \%$. This observation was consistent with a previous report showing that the uptake of the probe by EPCs is homogeneous. ${ }^{16}$

\section{Tracking bCD-PLL-labeled EPCs in ischemic hindlimbs}

Figure 3 shows the optical images of the mice recorded at different time points after intracardiac injection of
bCD-PLL-labeled EPCs and saline. A stronger fluorescence signal was detected in the ischemic hindlimbs of the mice treated with bCD-PLL-labeled EPCs at 1, 3, 5 and 7 days after intracardiac injection compared with the mice treated with saline (Figure $3 \mathrm{~A}$ and $\mathrm{B}$ ). The fluorescence signals in ex vivo images of the ischemic hindlimbs after removing the skin (Figure 3C) were like those obtained in vivo. Significantly, changes in the average signal and the 

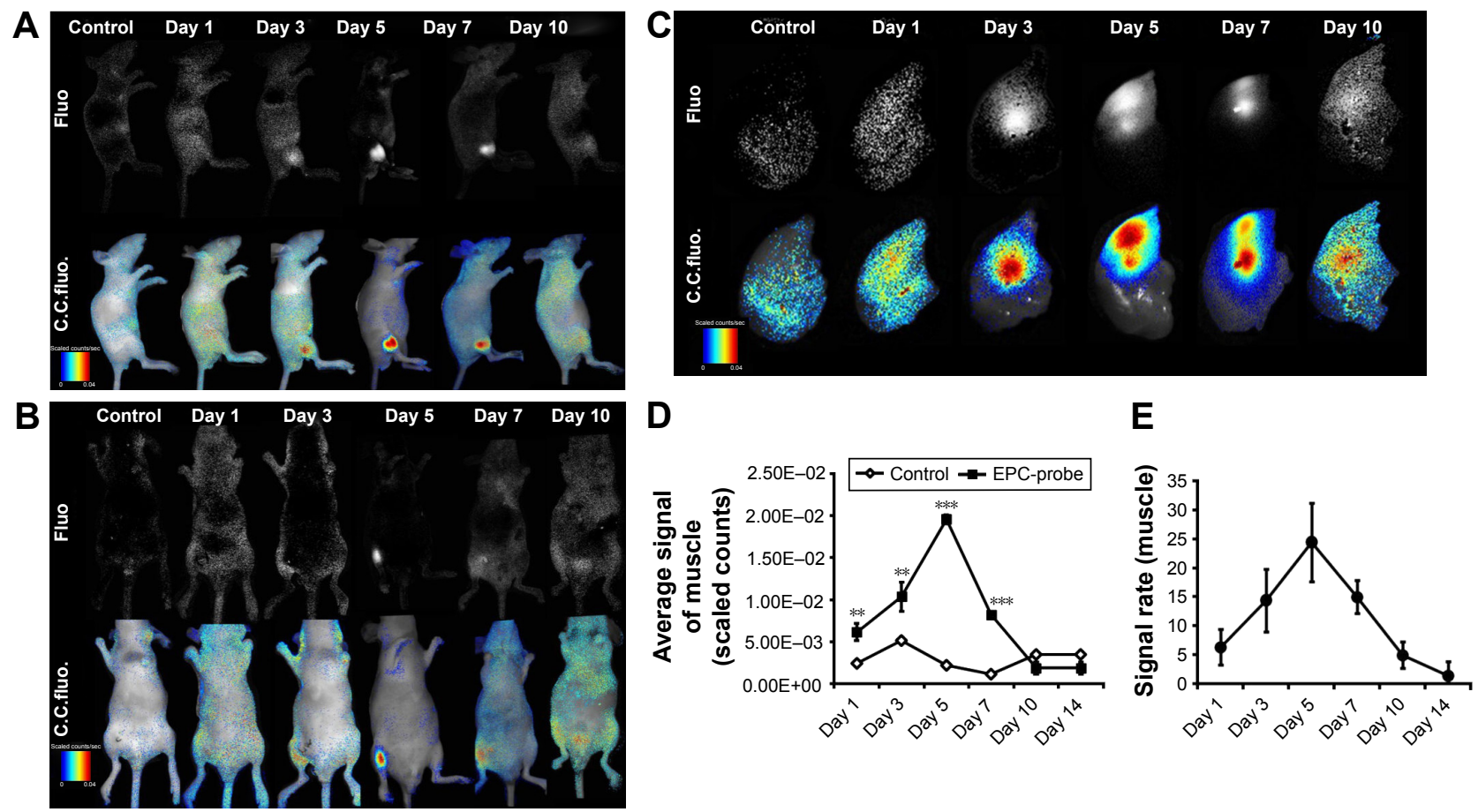

Figure 3 bCD-PLL-labeled EPCs observed in ischemic muscle using NIR optical imaging ( $\mathbf{A}$ and $\mathbf{B})$ in vivo (left-lateral and prone position) and (C) in vitro. Representative images showing that the signals observed in the ischemic hindlimbs on days I, 3, 5, 7 and 10 after injection of bCD-PLL-labeled EPCs were significantly stronger than in the control group. (D) The average signal levels in ischemic muscle and (E) the signal ratio (labeled EPCs/saline) were calculated from ROI analyses of the two groups. $* * P<0.0 \mathrm{I}$, $* * * P<0.001$

Abbreviations: bCD, bacterial cytosine deaminase; PLL, poly-L-lysine; EPC, endothelial progenitor cell; NIR, near infrared; ROI, region of interest; Fluo, fluorescence; C.C. fluo, color-coded fluorescence.

signal ratio in the hindlimb muscle were detected as early as day 1 after intracardiac injection of bCD-PLL-labeled EPCs. A time-dependent increase in the signal at the ischemic site was observed until day 7 after the injection of EPCs, with a peak signal detected on day 5 (Figure 3D and E), suggesting migration and homing of the EPCs. $\mathrm{T}_{1}$-weighted MRI revealed contrast enhancement at the periphery of the ischemic muscle on days 3 and 5 after the injection of bCD-PLL-labeled EPCs, while no contrast enhancement was observed in the group treated with saline. The average $T_{1}$ relaxation time of the ischemic muscle measured on days 3 and 5 in mice treated with bCD-PLL-labeled EPCs was significantly lower than in the control group (Figure 4A and $B$ ). This finding reflects the fact that the $T_{1}$ relaxation time of the ischemia tissue was shortened by the presence of $\mathrm{Gd}^{3+}$ in the bCD-PLL-labeled EPCs that migrated to the ischemic muscle. The time-dependent changes in Gd concentrations were determined using the ICP-MS analysis of homogenized tissue samples collected from hindlimb muscle with and without ischemia (Figure 4C). The Gd contents of ischemic muscle samples collected from mice treated with bCD-PLL-labeled EPCs on days 3, 5, 7, 10 and 14 after treatment were significantly higher than those of the nonischemic muscle samples.

\section{Tracking bCD-PLL-labeled EPCs in the liver and other organs}

The $T_{1}$ relaxation time of the liver in mice treated with bCD-PLL-labeled EPCs was also significantly lower than in the control group on days 3 and 5, suggesting some of the labeled EPCs accumulated in the liver (Figure 5A and B). The fluorescence signal of the liver in the group treated with labeled EPCs was also significantly higher on days 1, 3, 5, 7 and 10 after the injection of labeled EPCs (Figure 5C and D). The highest Gd concentrations in the liver, spleen and kidney were observed on day 5, consistent with the highest contrast enhancement observed from $\mathrm{T}_{1}$-weighted MRI (Figure 5F).

The Gd contents determined through ICP-MS in the samples from ischemic hindlimbs and livers also exhibited statistically significant linear correlations with the $\mathrm{T}_{1}$ relaxation times measured using MRI (Figure 6A) and the average fluorescence signal intensities recorded in the NIR images (Figure 6B) ( $\mathrm{T}_{1}$ relaxation time: $r=0.491$; average signal from optical imaging: $r=0.704, P<0.01)$.

Figure 7A and $\mathrm{B}$ shows that fluorescence signals from rhodamine were observed in tissue samples collected from mice treated with the bCD-PLL-labeled EPCs. These EPCs were double positive for CD34, CD133, CXCR4 or 


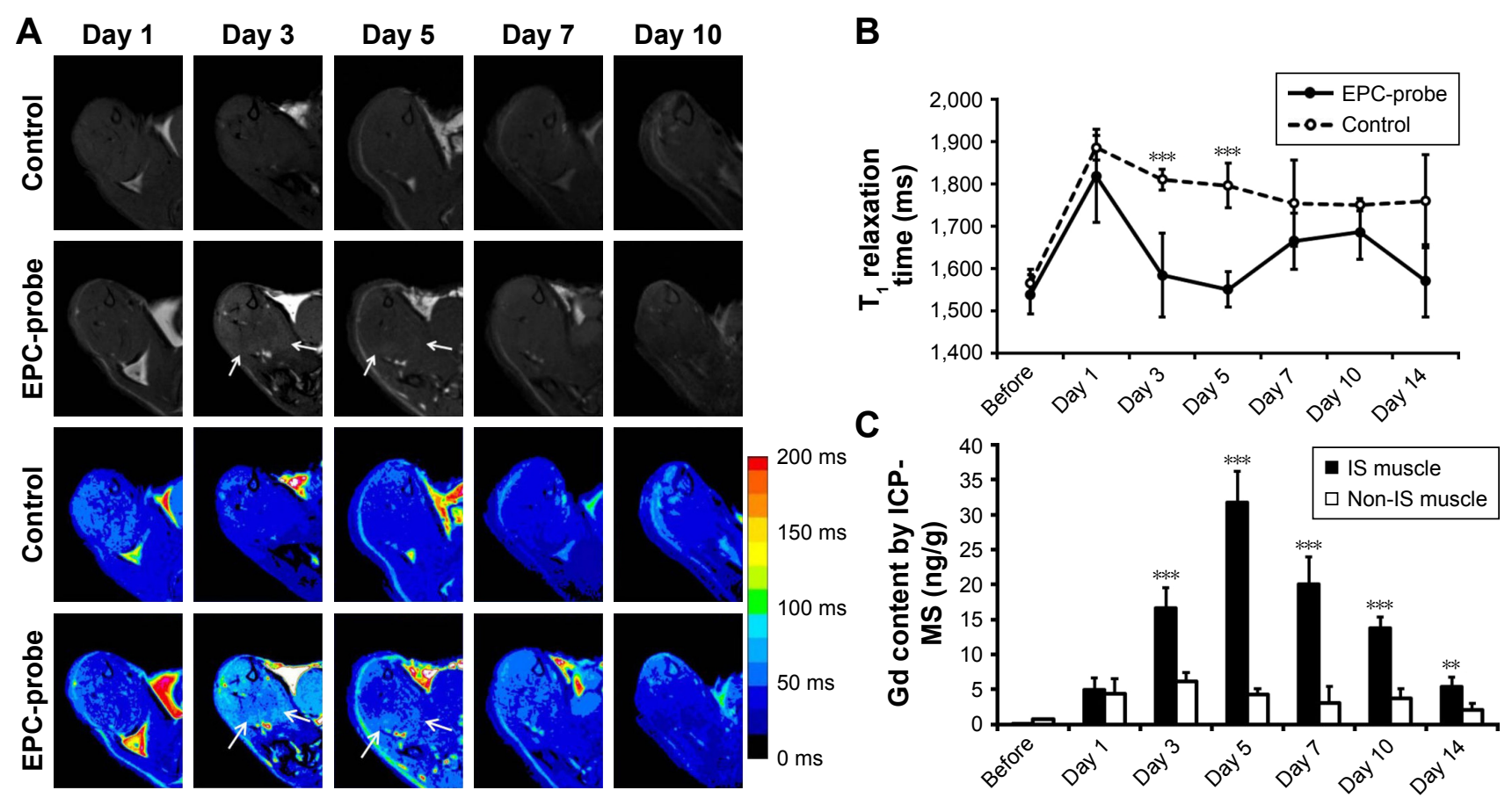

Figure 4 MRI tracking of bCD-PLL-labeled EPCs migrating to ischemic muscle and Gd concentrations measured via ICP-MS. (A) Representative T--weighted spin echo images with color-scaled signal intensities and (B) the $T_{\text {, }}$ relaxation times in hindlimb muscle observed at different times after the injection of bCD-PLL-labeled EPCs or control saline. The arrows indicate contrast enhancement at the periphery of ischemic muscle on days 3 and 5 after the injection of bCD-PLL-labeled EPCs. (C) Gd concentrations measured in ischemic and nonischemic muscle tissue via ICP-MS. $* * P<0.0$ I, $* * * P<0.00$ I.

Abbreviations: EPC, endothelial progenitor cell; MRI, magnetic resonance imaging; bCD, bacterial cytosine deaminase; PLL, poly-L-lysine; ICP-MS, inductively coupled plasma mass spectrometry; IS, ischemic.

VEGF receptor 2, further confirming the migration and homing of the probe-labeled EPCs to the injury site. The results of western blot analysis showed that the expression levels of CXCR4, SDF-1, VEGF receptor 2 and VEGF increased on days 1 and 3 after ischemic injury (Figure 7C). In addition, the expression levels of CXCR4, SDF-1, VEGF receptor 2 and VEGF in ischemic hindlimbs were significantly higher $(20 \%-50 \%)$ in the tissue samples collected from EPC-treated mice compared with those from control mice treated with saline (Figure 7D and E).

\section{Discussion}

MRI can be used as a noninvasive marker in vivo and as a diagnostic tool for the assessment of ischemia-induced muscle damage and repair. ${ }^{25}$ The results of a previous study suggested that ischemic tissue repair and muscle fiber regeneration were significantly improved after EPC treatment compared with a control group, based on in vivo MR diffusion tensor imaging. ${ }^{22}$ Although the repair mechanism of the transplanted EPCs remains unclear, the labeled bonemarrow-derived EPCs homed and migrated to the ischemic tissue after intracardiac injection, which was visualized using in vivo MRI and optical imaging. The results of this study demonstrated the feasibility of noninvasively tracking
EPCs through MRI and optical imaging in a mouse ischemic hindlimb model. Multimodal imaging also facilitates the evaluation of the migration of transplanted EPCs and therapeutic responses. However, our result showed the probe signal decreased after 7 days of probe-labeled EPCs transplantation and disappeared after 14 days. There are two major reasons for this phenomenon. First, with the cell division of probe-labeled EPCs, the intracellular probe signal decreased exponentially. Our previous study showed several rhodamine-positive endothelial cells after 7 days of ischemic stroke, suggesting that differentiation of the transplanted EPCs into mature endothelial cells had occurred. These results demonstrate that the transplanted EPCs were able to home to the ischemic area and incorporate into neovessels. ${ }^{24}$ Second, after probe-labeled EPCs died, intracellular probes were released and transported by inflammatory cells. ${ }^{26}$ The transplanted EPCs contributed to neovascularization either through incorporating into vessels or by secreting proangiogenic cytokines. ${ }^{27}$

The results showed that SDF-1 and VEGF expression significantly increased in ischemic muscle after EPC treatment compared with the controls on days 1 and 3. Our previous study demonstrated that EPC treatment promoted the expression of brain-derived neurotrophic factor and VEGF in 

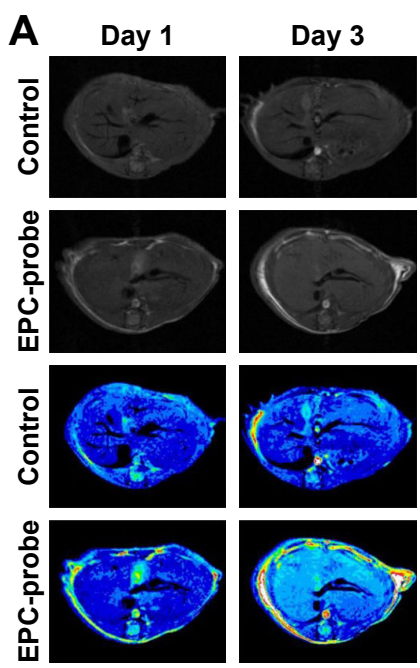

B

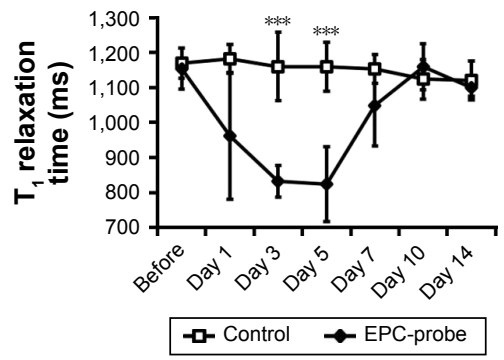

Day 5
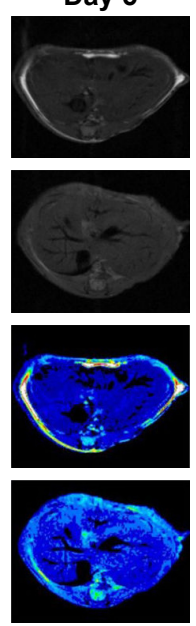

Day 7
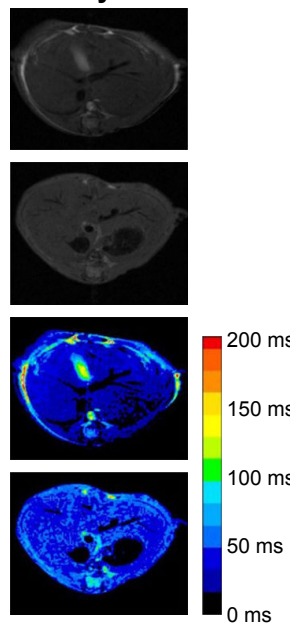

C

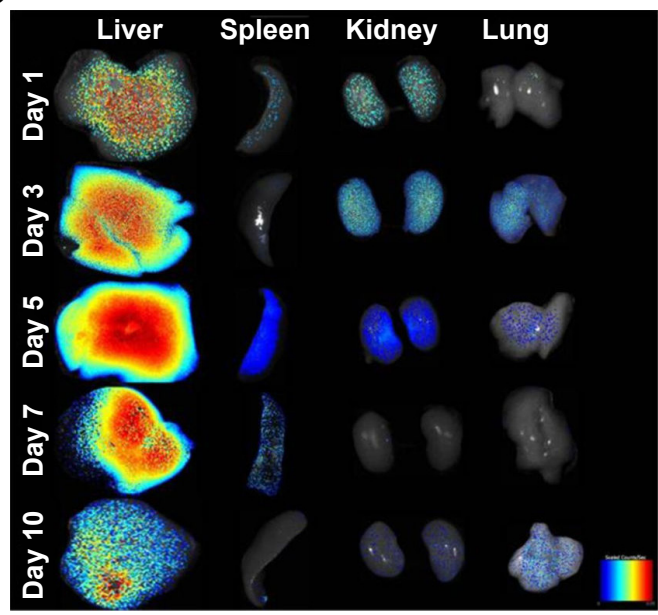

D

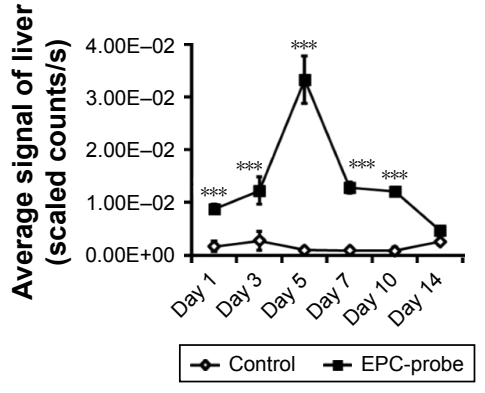

E
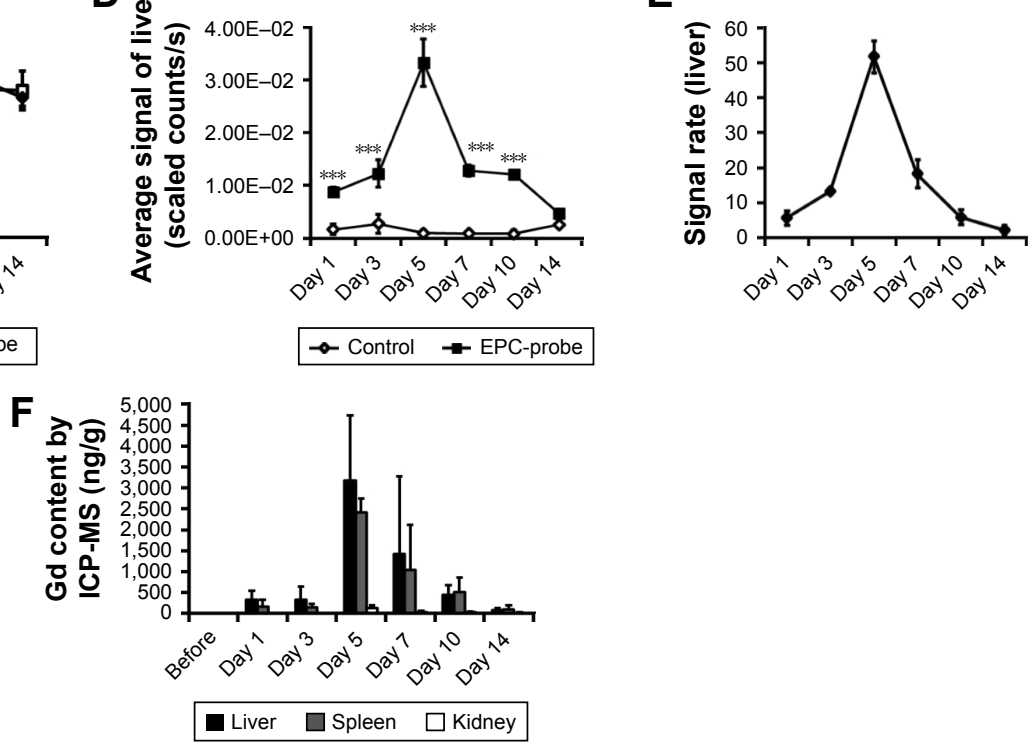

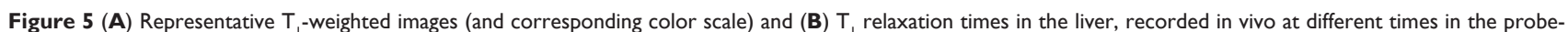
labeled EPC group or the control saline group. (C) In vitro optical imaging of the liver, spleen, kidneys and lungs at different time points after the transplantation of probelabeled EPCs showed an increase in the signal in the liver, spleen, kidneys and lungs. (D) The average signals in the liver and (E) the signal ratio were calculated from ROI analyses. (F) Gd concentrations measured via ICP-MS in the liver, spleen, kidneys and lungs. $* * * P<0.001$.

Abbreviations: EPC, endothelial progenitor cell; ROI, region of interest; ICP-MS, inductively coupled plasma mass spectrometry.

A

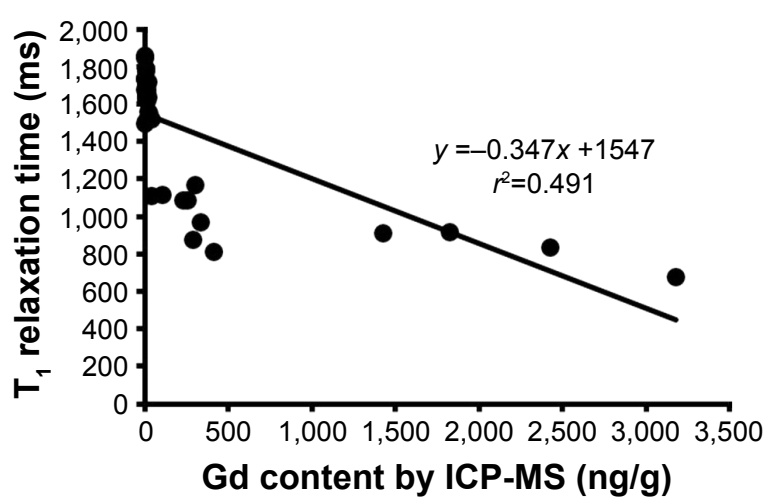

B

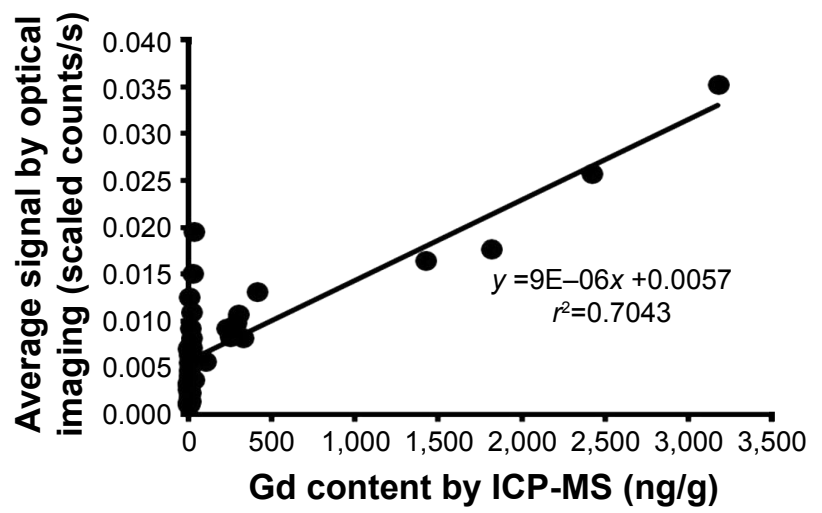

Figure 6 Scatter plots showing that the $(\mathbf{A}) T_{\text {, }}$ relaxation times $\left(r^{2}=0.491\right)$ and $(\mathbf{B})$ average signals from optical images $\left(r^{2}=0.704\right)$ of ischemic hindlimbs and livers are correlated with the Gd concentrations measured via ICP-MS in the same tissue samples.

Abbreviation: ICP-MS, inductively coupled plasma mass spectrometry. 
A
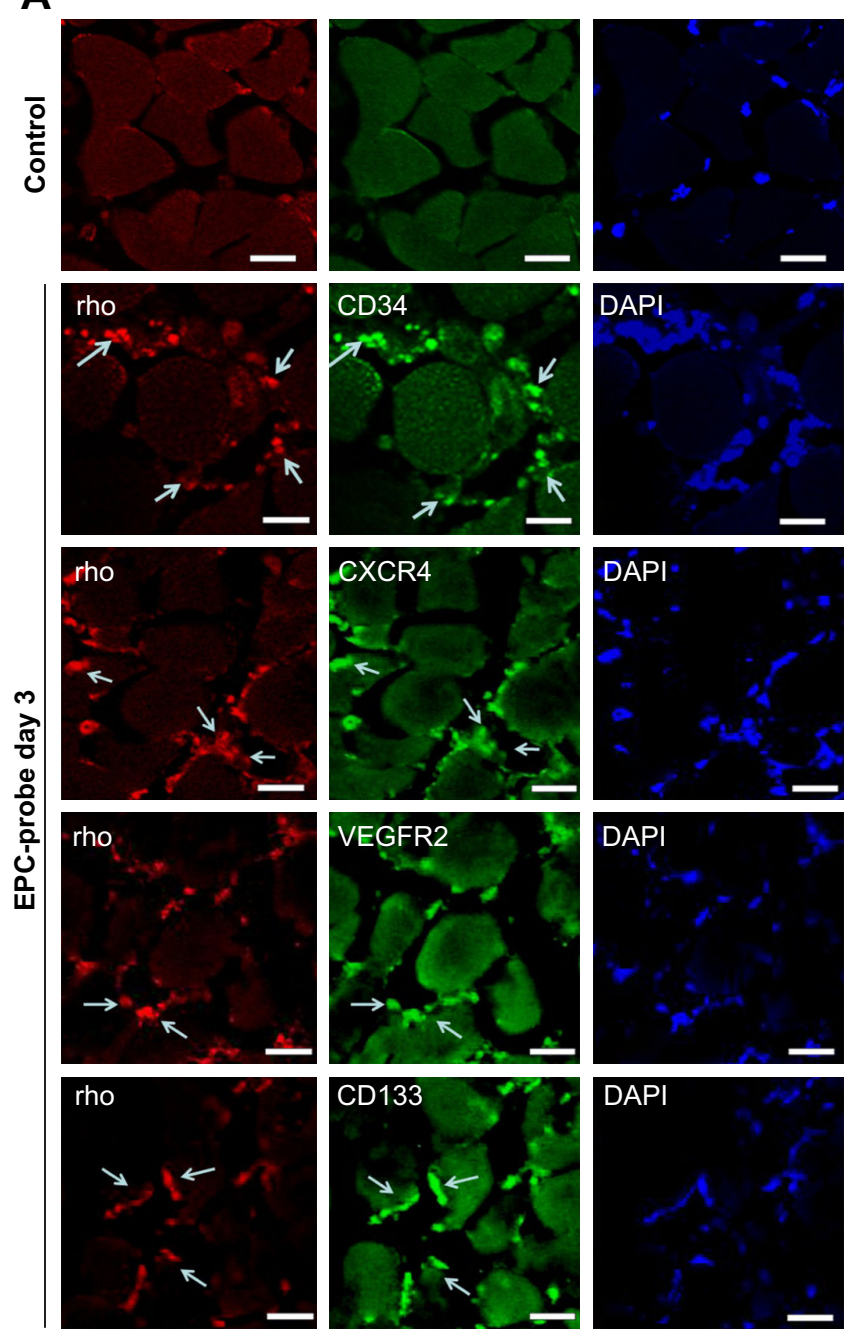
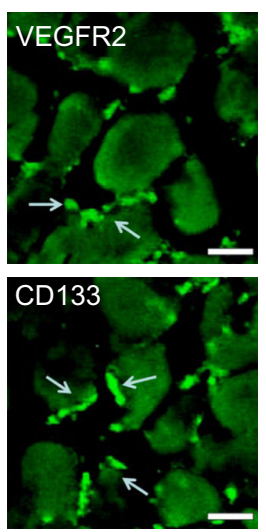
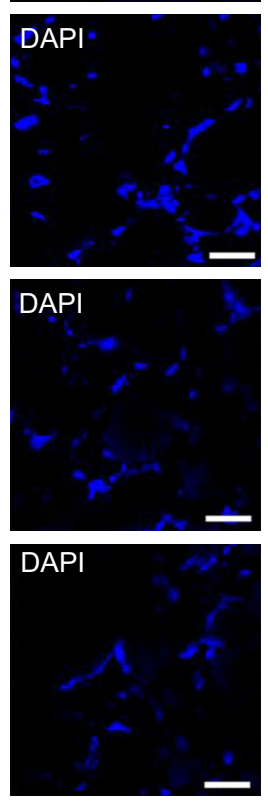
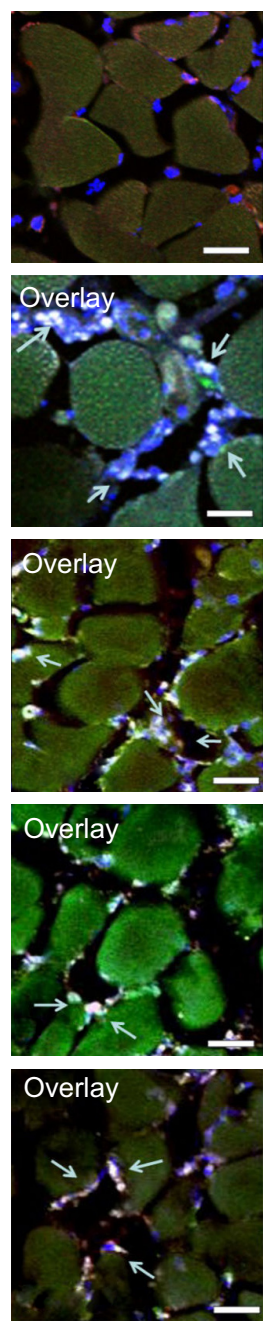

B

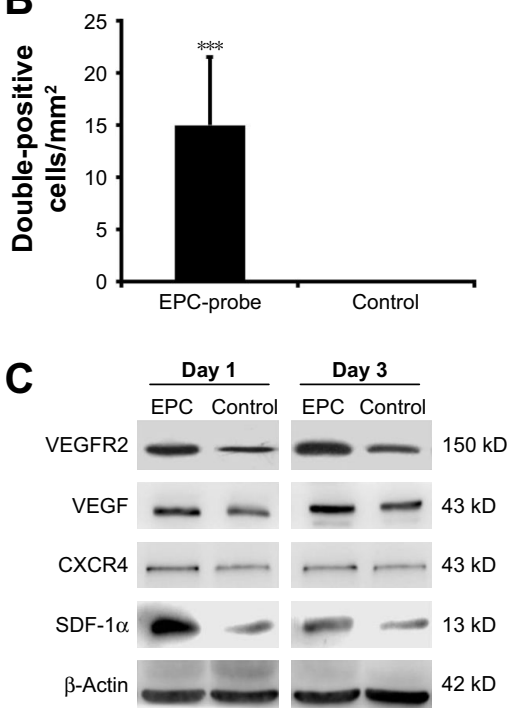

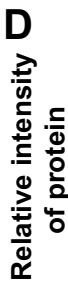
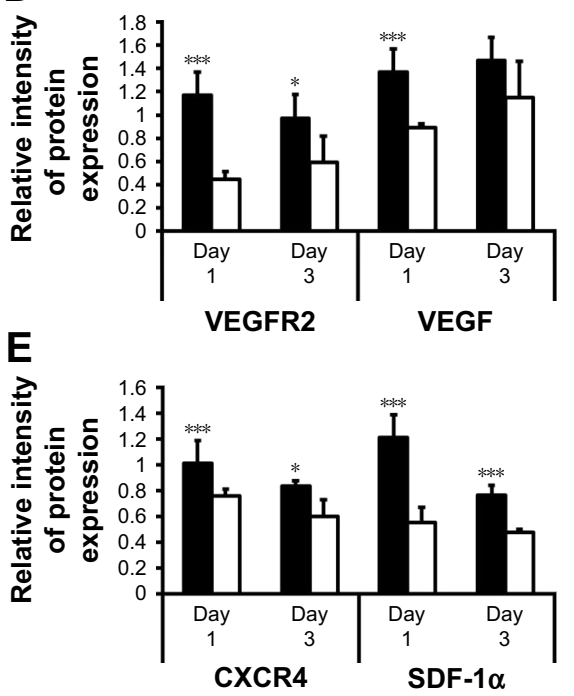

- EPC $\square$ Control

Figure 7 Detection of EPCs labeled with multimodal imaging probes in ischemic hindlimbs. (A) Cy5.5 and random signals from probe-labeled EPCs were colocalized in the injured tissue stained with fluorescent Alexa488-CD34, CDI33, VEGF receptor 2 and CXCR4 (EPC markers). Probe-labeled EPCs positive for EPC markers migrated into microvessel walls 3 days after transplantation. Cy5.5 and EPC antibody positive cells are shown by arrows. The scale bars are $30 \mu \mathrm{m}$. (B) The numbers of Cy5.5-positive and rhodamine double-positive EPCs per square millimeter within the ischemic region were recorded at 3 days after the induction of injury. (C) Western blot showing increased VEGF receptor 2, VEGF, CXCR4 and SDF-I $\alpha$ levels in the ischemic muscle of EPC-treated mice on days I and 3 compared with the controls. (D) and (E) Bottom panel shows the results of quantification of the bands. $* P<0.05$, $* * * P<0.001$

Abbreviations: EPC, endothelial progenitor cell; VEGF, vascular endothelial growth factor; CXCR4, C-X-C chemokine receptor 4; SDF-I $\alpha$, stromal cell-derived factorI alpha; rho, rhodamine; DAPI, 4',6-diamidino-2-phenylindole; VEGFR2, VEGF receptor 2.

ischemic stroke mice. ${ }^{24,28}$ VEGF not only promotes endothelial cell proliferation, including angiogenesis but also promotes the growth of myogenic fibers and protects myogenic cells from apoptosis. ${ }^{29,30}$ In addition, CXCR4 and VEGF receptor 2 were upregulated in ischemic muscle in the EPC-treated group compared with the control mice. The SDF-1-CXCR4 axis is critical for tissue repair in multiple organ systems, including the skin, brain and heart. ${ }^{31-35}$ Overexpression of SDF-1 improves tissue repair by recruiting tissue-specific and bone marrow-derived stem cells. ${ }^{36}$ The results of the present study suggested that EPCs transplantation stimulated the recruitment of proangiogenic monocytes through EPC activation and enhanced chemotaxis in ischemic muscle.

Low rates of cell homing, retention and survival are currently the major limitations in the development of cell therapies using EPCs. Until recently, two strategies have been used to augment cell engraftment. The first approach involves intravascular delivery of EPCs, which may spontaneously extravasate and migrate to the target tissue. In the second approach, the cells or target tissues are pretreated to increase 
cell homing and survival. ${ }^{37}$ In the present study, intracardiac injection of late bone morrow-derived EPCs exerted a positive arteriogenic effect on ischemic tissue. However, Gianella et al previously suggested that intramuscular injection of early EPCs labeled with iron-based contrast agents elicits a longterm inflammatory response that reflects a delayed muscle healing process. ${ }^{26}$ In contrast, Frederick et al demonstrated that the implantation of an engineered extracellular matrix seeded with EPCs primed with SDF-1 induces border zone neovasculogenesis, attenuates adverse ventricular remodeling and preserves ventricular function after myocardial infarction. ${ }^{38}$ The inconsistencies among these studies, including the present study, suggest that the methods of EPC transplantation and the local environment of EPC transplantation could influence cell survival and the effect of therapy. Nevertheless, intracoronary cell therapy following percutaneous coronary intervention for acute myocardial infarction has been shown to provide statistically and clinically relevant benefits to cardiac function and remodeling. ${ }^{39}$ In addition, it should be taken into account that athymic mice are deficient in T-cell responses but harbor other inflammatory cells, including natural killer cells and other cells involved in the innate immune response. ${ }^{40}$ Our previous study showed by ICP-MS that the Gd content of ischemic brain tissue in normal mice (C57BLKS/J) model after probe-labeled EPCs treatment was about $700 \mathrm{ng} / \mathrm{g} .{ }^{24}$ However, another previous study by our group demonstrated that the Gd content of breast tumor tissue in nude mice after probe-labeled EPCs treatment was only about $60 \mathrm{ng} / \mathrm{g} .{ }^{18}$ This present study also displayed the same result that very lower Gd content of ischemic muscle tissue was present in nude mice (about $35 \mathrm{ng} / \mathrm{g}$ ). Thus, transplanted EPCs could be degraded by the immune system, which leads to decrease in the amount of immigrated EPCs. This is a possibility that requires further investigation in the future.

In summary, we showed that bone marrow-derived EPCs labeled with a multimodal imaging probe administered by intracardiac injection could be tracked in vivo and monitored in vitro in a murine ischemic hindlimb model using MRI and optical imaging.

\section{Abbreviations}

acLDL, acetylated low-density lipoprotein; bCD, bacterial cytosine deaminase; CXCR4, C-X-C chemokine receptor 4; DOTA, tetraazacyclododecane-1,4,7,10-tetraacetic acid; EPC, endothelial progenitor cell; FOV, field of view; ICP-MS, inductively coupled plasma mass spectrometry; MRI, magnetic resonance imaging; NIR, near infrared; PBS, phosphate buffered saline; PLL, poly-L-lysine; ROI, region of interest; SDF-1, stromal cell-derived factor-1; TE, echo time; TR, repetition time; UEA-1, Ulex europaeus agglutinin-1; VEGF, vascular endothelial growth factor; vWF, von Willebrand factor.

\section{Acknowledgments}

This work was financially supported by grants from the National Natural Science Foundation of China (No. 81501523, No. 81525014, No. 81371538), the Jiangsu Provincial Special Program of Medical Science (BL2013029) and the Jiangsu Provincial Science and Technology Program (BK20151423).

\section{Disclosure}

The authors report no conflicts of interest in this work.

\section{References}

1. Yusuf S, Reddy S, Ôunpuu S, Anand S. Global burden of cardiovascular diseases: Part II: variations in cardiovascular disease by specific ethnic groups and geographic regions and prevention strategies. Circulation. 2001;104(23):2855-2864.

2. Asahara T, Murohara T, Sullivan A, et al. Isolation of putative progenitor endothelial cells for angiogenesis. Science. 1997;275(5302):964-967.

3. Asahara T, Masuda H, Takahashi T, et al. Bone marrow origin of endothelial progenitor cells responsible for postnatal vasculogenesis in physiological and pathological neovascularization. Circ Res. 1999; 85(3):221-228.

4. Takahashi T, Kalka C, Masuda H, et al. Ischemia- and cytokine-induced mobilization of bone marrow-derived endothelial progenitor cells for neovascularization. Nat Med. 1999;5(4):434-438.

5. Sieveking DP, Ng MKC. Cell therapies for therapeutic angiogenesis: back to the bench. Vasc Med. 2009;14(2):153-166.

6. Alev C, Ii M, Asahara T. Endothelial progenitor cells: a "novel" tool for the therapy of ischemic diseases. Antioxid Redox Signal. 2011; 15(4):949-965.

7. Asahara T, Kawamoto A, Masuda H. Concise review: circulating endothelial progenitor cells for vascular medicine. Stem Cells. 2011; 29(11):1650-1655.

8. Bautch VL. Stem cells and the vasculature. Nat Med. 2011;17(11): 1437-1443.

9. Iwakura A, Shastry S, Luedemann C, et al. Estradiol enhances recovery after myocardial infarction by augmenting incorporation of bone marrow-derived endothelial progenitor cells into sites of ischemiainduced neovascularization via endothelial nitric oxide synthasemediated activation of matrix metalloproteinase-9. Circulation. 2006; 113(12):1605-1614.

10. Yao L, Heuser-Baker J, Herlea-Pana O, et al. Bone marrow endothelial progenitors augment atherosclerotic plaque regression in a mouse model of plasma lipid lowering. Stem Cells. 2012;30(12):2720-2731.

11. Bauer SM, Goldstein LJ, Bauer RJ, Chen H, Putt M, Velazquez OC. The bone marrow-derived endothelial progenitor cell response is impaired in delayed wound healing from ischemia. J Vasc Surg. 2006; 43(1):134-141.

12. Masuda H, Kalka C, Takahashi T, et al. Estrogen-mediated endothelial progenitor cell biology and kinetics for physiological postnatal vasculogenesis. Circ Res. 2007;101(6):598-606.

13. Vahrmeijer AL, Hutteman M, van der Vorst JR, van de Velde CJ, Frangioni JV. Image-guided cancer surgery using near-infrared fluorescence. Nat Rev Clin Oncol. 2013;10(9):507-518. 
14. Wang C, Tao H, Cheng L, Liu Z. Near-infrared light induced in vivo photodynamic therapy of cancer based on upconversion nanoparticles. Biomaterials. 2011;32(26):6145-6154.

15. Yao Y, Li Y, Ma G, et al. In vivo magnetic resonance imaging of injected endothelial progenitor cells after myocardial infarction in rats. Mol Imaging Biol. 2011;13(2):303-313.

16. Ju S, Qiu Y, Li C, Teng GJ, Ni Y. Multimodality imaging of endothelial progenitor cells with a novel multifunctional probe featuring positive magnetic resonance contrast and near-infrared fluorescence. Mol Imaging. 2011;10(5):359-369.

17. Ju S, Teng G, Zhang Y, Ma M, Chen F, Ni Y. In vitro labeling and MRI of mesenchymal stem cells from human umbilical cord blood. Magn Reson Imaging. 2006;24(5):611-617.

18. Wang XY, Ju S, Li C, et al. Non-invasive imaging of endothelial progenitor cells in tumor neovascularization using a novel dual-modality paramagnetic/near-infrared fluorescence probe. PLoS One. 2012;7(11): e50575.

19. Li C, Wildes F, Winnard P Jr, Artemov D, Penet M-F, Bhujwalla ZM. Conjugation of poly-1-lysine to bacterial cytosine deaminase improves the efficacy of enzyme/prodrug cancer therapy. J Med Chem. 2008; 51(12):3572-3582.

20. Li C, Winnard PT Jr, Takagi T, Artemov D, Bhujwalla ZM. Multimodal image-guided enzyme/prodrug cancer therapy. J Am Chem Soc. 2006;128(47):15072-15073.

21. Chen R, Yu H, Jia Z, Yao QL, Teng GJ. Efficient nano iron particlelabeling and noninvasive MR imaging of mouse bone marrowderived endothelial progenitor cells. Int J Nanomedicine. 2011;6: 511-519.

22. Peng XG, Bai YY, James JR, Shlapak DP, Ju S. Transplanted endothelial progenitor cells improve ischemia muscle regeneration in mice by diffusion tensor MR imaging. Stem Cells Int. 2016;2016:3641401.

23. Zhang Y, Fan S, Yao Y, et al. In vivo near-infrared imaging of fibrin deposition in thromboembolic stroke in mice. PLoS One. 2012;7(1): e30262.

24. Bai YY, Wang L, Peng XG, et al. Non-invasive monitoring of transplanted endothelial progenitor cells in diabetic ischemic stroke models. Biomaterials. 2015;40(43):43-50.

25. Heemskerk AM, Strijkers GJ, Drost MR, van Bochove GS, Nicolay K. Skeletal muscle degeneration and regeneration after femoral artery ligation in mice: monitoring with diffusion MR imaging. Radiology. 2007;243(2):413-421.

26. Gianella A, Guerrini U, Tilenni M, et al. Magnetic resonance imaging of human endothelial progenitors reveals opposite effects on vascular and muscle regeneration into ischaemic tissues. Cardiovasc Res. 2010; 85(3):503-513.

27. Urbich C, Dimmeler S. Endothelial progenitor cells: characterization and role in vascular biology. Circ Res. 2004;95(4):343-353.
28. Bai YY, Peng XG, Wang LS, et al. Bone marrow endothelial progenitor cell transplantation after ischemic stroke: an investigation into its possible mechanism. CNS Neurosci Ther. 2015;21(11):877-886.

29. Arsic N, Zacchigna S, Zentilin L, et al. Vascular endothelial growth factor stimulates skeletal muscle regeneration in vivo. Mol Ther. 2004; 10(5):844-854.

30. Rissanen TT, Vajanto I, Hiltunen MO, et al. Expression of vascular endothelial growth factor and vascular endothelial growth factor receptor-2 (KDR/Flk-1) in ischemic skeletal muscle and its regeneration. Am J Pathol. 2002;160(4):1393-1403.

31. Zhang M, Mal N, Kiedrowski M, et al. SDF-1 expression by mesenchymal stem cells results in trophic support of cardiac myocytes after myocardial infarction. FASEB J. 2007;21(12):3197-3207.

32. Lee JS, Hong JM, Moon GJ, Lee PH, Ahn YH, Bang OY. A long-term follow-up study of intravenous autologous mesenchymal stem cell transplantation in patients with ischemic stroke. Stem Cells. 2010;28(6): 1099-1106.

33. Penn MS. SDF-1:CXCR4 axis is fundamental for tissue preservation and repair. Am J Pathol. 2010;177(5):2166-2168.

34. Dong F, Harvey J, Finan A, Weber K, Agarwal U, Penn MS. Myocardial CXCR4 expression is required for mesenchymal stem cell mediated repair following acute myocardial infarction. Circulation. 2012; 126(3):314-324.

35. Hu X, Dai S, Wu WJ, et al. Stromal cell-derived factor-1 $\alpha$ confers protection against myocardial ischemia/reperfusion injury. Circulation. 2007;116(6):654-663.

36. Tang J, Wang J, Yang J, et al. Mesenchymal stem cells over-expressing SDF-1 promote angiogenesis and improve heart function in experimental myocardial infarction in rats. Eur J Cardiothorac Surg. 2009; 36(4):644-650.

37. Chavakis E, Koyanagi M, Dimmeler S. Enhancing the outcome of cell therapy for cardiac repair: progress from bench to bedside and back. Circulation. 2010;121(2):325-335.

38. Frederick JR, Fitzpatrick JR III, McCormick RC, et al. Stromal cell-derived factor- $1 \alpha$ activation of tissue-engineered endothelial progenitor cell matrix enhances ventricular function after myocardial infarction by inducing neovasculogenesis. Circulation. 2010; 122(11 Suppl 1):S107-S117.

39. Vetrovec GW, Strauer BE, Kang HJ, et al. Impact of intracoronary cell therapy on left ventricular function in the setting of acute myocardial infarction: a collaborative systematic review and meta-analysis of controlled clinical trials. J Am Coll Cardiol. 2007;50(18):1761-1767.

40. Pelleitier M, Montplaisir S. The nude mouse: a model of deficient T-cell function. Methods Achiev Exp Pathol. 1975;7:149-166.
International Journal of Nanomedicine

\section{Publish your work in this journal}

The International Journal of Nanomedicine is an international, peerreviewed journal focusing on the application of nanotechnology in diagnostics, therapeutics, and drug delivery systems throughout the biomedical field. This journal is indexed on PubMed Central, MedLine, CAS, SciSearch $®$, Current Contents $\AA /$ Clinical Medicine,

\section{Dovepress}

Journal Citation Reports/Science Edition, EMBase, Scopus and the Elsevier Bibliographic databases. The manuscript management system is completely online and includes a very quick and fair peer-review system, which is all easy to use. Visit http://www.dovepress.com/ testimonials.php to read real quotes from published authors. 